\title{
Aprendizaje Basado en Problemas en Currículos Tradicionales: Beneficios e Inconvenientes
}

\author{
Itxaro Latasa ${ }^{(1)}$, Peio Lozano ${ }^{(1)}$ y Nagore Ocerinjauregi ${ }^{(2)}$ \\ (1) Universidad del País Vasco (UPV/EHU), Departamento de Geografía, Prehistoria y \\ Arqueología. Paseo de la Universidad, 5. 01006 Vitoria-Gasteiz-España. \\ (e-mail: itxaro.latasa@ehu.es) \\ (2) Universidad del País Vasco (UPV/EHU. Departamento de Didáctica y Organización Escolar, \\ Escuela Universitaria de Magisterio de Vitoria-Gasteiz. Juan Ibáñez de Sto. Domingo, 1 \\ 01006 Vitoria-Gasteiz.-España
}

Recibido Abr. 16, 2012; Aceptado May. 16, 2012; Versión final recibida Jun. 26, 2012

\begin{abstract}
Resumen
El artículo expone los objetivos, la metodología y los resultados de una investigación destinada a evaluar los efectos en el aprendizaje derivados de la utilización de un módulo de Aprendizaje Basado en Problemas (ABP). Se diseñó un programa formativo basado en las propuestas teóricas del ABP y adaptado a las características particulares de los estudiantes habituales del primer curso del grado en Geografía y Ordenación del Territorio, principiantes universitarios escasamente capacitados para el aprendizaje autónomo. Los resultados obtenidos muestran que la utilización del módulo ABP ha producido mejoras significativas en aspectos fundamentales del aprendizaje como la motivación, la capacidad de procesamiento de la información y el grado de colaboración entre los estudiantes. La experiencia ha mostrado también que existen inconvenientes y obstáculos que comprometen la viabilidad de la estrategia metodológica empleada.
\end{abstract}

Palabras clave: enseñanza-aprendizaje, aprendizaje basado en problemas, currículo tradicional, principiantes universitarios, aprendizaje autónomo

\section{Problem-Based Learning within Traditional Curricula : Benefits and Inconveniences}

\begin{abstract}
The paper sets out the objectives, the methodology and the results of a research project related to the effects in the learning process of students produced by the use of a module of Problem Based Learning (PBL). A teaching programme based in the theoretical foundations of PBL and adapted to the particular characteristics of students of the first year in Geography and Land Planning was designed. The recipients of the teaching programme are freshmen students hardly capable of self learning. The results show that the use of the PBL module in the class has produced significant improvements in fundamental aspects of the learning process such as motivation, capacity of information processing and the degree of collaboration among students. The experience has also shown that inconveniences and obstacles the threat the viability of the methodological strategy used do exist.
\end{abstract}

Keywords: teaching-learning, problem based learning, traditional curriculum, university beginners, self-directed learning. 


\section{INTRODUCCION}

La transformación del modelo universitario español, ligada fundamentalmente a la creación de Espacio Europeo de Educación Superior ha supuesto la realización de cambios de diverso tipo y calado en la estructura y modos de funcionamiento de la entidad universitaria. De todos estos cambios, sin duda, el más importante y costoso -y el más demandado- es el que afecta a la práctica docente del profesorado universitario, que debe estar basada en la enseñanza y más en el aprendizaje (Casado Ortiz, 2006, Michavila, 2009, Martínez Román, 2009). Tanto es así que el Ministerio de Educación y Ciencia propuso la creación de una Comisión para la Renovación de las Metodologías Educativas, que se constituyó en 2005. Un año más tarde se publicó el informe final de la comisión (MEC, 2006), una de cuyas conclusiones fundamentales alude a la necesidad de cambiar los métodos de enseñanza y aprendizaje de la universidad española.

Cuando han pasado casi dos años ya desde que culminó el proceso de convergencia hacia el Espacio Europeo de Educación Superior, la cuestión es, como se pregunta Michavila (2009), si han cambiado mucho los procesos de formación de los titulados universitarios en los tiempos recientes. La situación sigue siendo, para este autor, todavía bastante pesimista. Predomina, por el momento, la formación centrada en el profesor, apoyada por el hecho de que para la mayoría de los estudiantes que acceden a la Universidad, el aula no es un espacio para aprender, sino para recoger la información que contará o proporcionará el profesor. Aunque son pocos los que se atreven a hacer afirmaciones tan rotundas, son muchos los que hablan en términos de obstáculos y dificultades de la reforma como consecuencia de la falta de dotación económica o de la complejidad de los cambios estructurales e instrumentales que exige (Sáez Pérez, 2008; Morales Gil et al. 2010).

A falta de un cambio legislativo profundo y de directrices operativas suficientes para acometer la reforma metodológica (Sáez Pérez, 2008), las iniciativas han quedado en manos de los ICEs (Institutos de Ciencias de la Educación) y de los servicios pedagógicos adscritos a las universidades españolas. Estas entidades han organizado numerosos congresos, seminarios y actividades diversas en relación con las nuevas funciones docentes y las metodologías activas de enseñanza-aprendizaje. Se han encargado también de ofertar diversos programas y cursos de formación para el profesorado universitario. Este es el modelo seguido por la Universidad del País Vasco (UPV/EHU) desde cuyo Servicio de Orientación Universitaria se ha creado un programa de trabajo destinado a impulsar la transformación del modelo formativo. Ahora bien, como en la mayoría de universidades españolas, las acciones emprendidas se limitan a la formación del profesorado.

Debido a la falta de un marco institucional regulador, la incorporación de metodologías activas de enseñanza-aprendizaje en la Universidad del País Vasco se produce, necesariamente, a través de experiencias docentes individuales insertas en currículos tradicionales, estructurados en base a asignaturas temáticas. Este hecho supone una limitación para la puesta en práctica de métodos que requieren un enfoque interdisciplinar integrado y una estrecha coordinación entre el profesorado. Cuando estas condiciones no se cumplen, es preciso preguntarse si la aplicación de tales métodos puede reportar mejoras significativas en el aprendizaje. Esta es, precisamente, la pregunta que da origen a nuestra investigación y que surge como consecuencia de la voluntad de la UVP de incorporar el ABP como metodología de enseñanza-aprendizaje en los primeros cursos de las nuevas titulaciones adaptadas al EEES.

Pese a la enorme popularidad del Aprendizaje Basado en Problemas, de los beneficios que se le atribuyen y de que se ha extendido a numerosos ámbitos de la educación, hoy en día no existe un consenso unánime en relación a los fundamentos teóricos ni a su práctica (Greening, 1998; Maudsley, 1999; Dochy et al 2003; Newman, 2005; Fernández et al., 2006), hasta el punto de que, como señala Newman (2005), existen en la literatura sobre el tema posiciones contradictorias sobre los rasgos fundamentales de este enfoque. Ante la diversidad de propuestas existentes sobre lo que es o debe ser el ABP, algunos autores intentan recuperar la identidad del ABP original, para lo cual defienden como esenciales las características del que consideran el verdadero ABP o su forma más pura (Albanese, 2010). Dentro de esta línea, Maudsley (1999) y 
Greening (1998) son taxativos con respecto a la esencia del verdadero ABP: se trata de una estrategia formativa que abarca la totalidad del currículo y no simplemente un método. El uso del $A B P$, por tanto, exige un currículo integrado, como requisito para que se puedan crear las condiciones de un aprendizaje activo, iterativo y autodirigido, que se produce fundamentalmente en un contexto de complejidad progresiva no constreñido por las limitaciones de un currículo de estructura temática. Desde la perspectiva planteada resultaría imposible utilizar el ABP en cursos aislados. De hecho, Maudsley (1999) afirma que muchos de los cursos pretendidamente ABP que se imparten dentro de currículos tradicionales no usan en absoluto este enfoque. Para comprender esta afirmación es preciso entender que no se trata de una cuestión puramente semántica, sino que, como señala Newman (2005), el problema se relaciona con la validez de los resultados obtenidos bajo condiciones muy diferentes de aplicación del ABP.

Otros autores, sin embargo, no dudan de la validez de los resultados obtenidos mediante la aplicación del ABP en contextos no integrados. Dochy et al. (2003) como resultado de un metaanálisis estadístico de los resultados de 43 investigaciones empíricas sobre los efectos del ABP, no encontraron diferencias significativas entre los resultados del ABP aplicado en todo el currículo y los resultados del ABP aplicado en cursos individuales. En ambos casos se constatan efectos positivos claros. Sobral (1995), en su artículo Peer tutoring and student outcomes in a problembased course, concluye que la utilización del ABP, incluso en cursos individuales, puede mejorar el bienestar emocional de los participantes y la calidad del entorno de aprendizaje. (Greening, 1988).

Por otro lado, es fácil comprobar que el ABP se está usando cada vez más como técnica didáctica en cursos individuales dentro de currículos tradicionales (Albanese, 2010; Fernández Martínez et al., 2006, Lee et al., 2006, Levia y Quiring, 2008, Mitchell y Smith, 2008). Los beneficios que se le atribuyen, el deseo de mejorar la práctica docente e innovar en la enseñanza (Lobato y Medinabeitia, 2011) o, quizás, simplemente la aceptación que suscita el ABP nos hacen olvidar los requisitos a los que aludíamos anteriormente. Sea como fuere, la situación suscita numerosos interrogantes. ¿Cómo es posible abordar una estrategia de enseñanza-aprendizaje basada en el trabajo autónomo cuando los estudiantes universitarios españoles, en general, carecen de esta capacidad? (Martínez Román et al., 2010); ¿De qué modo se puede o se debe organizar la actividad docente?, ¿qué resultados se están obteniendo de la aplicación del ABP en cursos individuales?, ¿qué valor tienen las evidencias encontradas?

Teniendo en cuenta que el ABP (el verdadero $A B P$ ) se plantea como un enfoque integral y que la mayoría de las investigaciones sobre su efectividad se realizan en este contexto, hoy en día todavía existen muy pocas evidencias procedentes de investigación de alta calidad sobre la efectividad del ABP aplicado de forma aislada dentro de currículos tradicionales. Lee et al. (2009) califican de inconsistentes las evidencias existentes en este ámbito de la investigación, fundamentalmente en lo referente a los efectos del ABP en la mejora del aprendizaje autodirigido. Consecuentemente, no existe una base de conocimiento sólida y contrastada sobre las posibles particularidades del uso del ABP en experiencias aisladas.

La situación expuesta nos conduce a dos conclusiones. Una de ellas es que existen dudas razonables sobre los beneficios que puede producir la utilización del ABP en situaciones de aprendizaje aisladas. Esta carencia anima la investigación en este ámbito. La segunda de ellas se refiere al hecho de que cualquier intento de utilizar el enfoque ABP en condiciones distintas a las que proporciona un currículo ABP integrado, debe partir de un análisis sobre la especificidad de las condiciones en las que se va a aplicar la experiencia $y$, fundamentalmente, sobre las posibilidades y limitaciones de la aplicación del ABP al caso concreto. Consideramos que es fundamental definir los objetivos a los que podemos aspirar y aquellos a los que, quizás, debemos renunciar porque dichos objetivos se alcanzan bajo condiciones de enseñanza-aprendizaje que no podemos aplicar. En cualquier caso, lo esencial es que seamos extremadamente rigurosos a la hora de especificar los rasgos del modelo que estamos aplicando, los objetivos que perseguimos $y$, fundamentalmente, los procedimientos para evaluar los resultados. 
En nuestro caso, se diseñó un módulo ABP para la asignatura de Cartografía Básica. Se trata de una asignatura de carácter obligatorio que se imparte durante el primer cuatrimestre del primer curso de la titulación de Geografía y Ordenación del Territorio en la Universidad del País Vasco UPV/EHU. El diseño del módulo ABP se apoyó en primer lugar en una experiencia docente de 16 años con grupos de alumnos que acceden a la titulación. Respecto a esta experiencia, es preciso señalar que, aunque a lo largo de los últimos años los autores hemos puesto en práctica estrategias y enfoques variados destinados a impulsar el aprendizaje autodirigido y las actividades colaborativas, es esta la primera vez que utilizamos el enfoque ABP en nuestra docencia. En cualquier caso, esta experiencia ha constituído la base para establecer una serie de hipótesis iniciales sobre las capacidades, los hábitos y las técnicas de estudio esperables en los alumnos a los que se iba a aplicar el módulo ABP y que sirvieron para realizar un diseño inicial del módulo. Un cuestionario realizado a los alumnos el primer día del curso nos permitió corroborar y matizar las hipótesis iniciales y completar el diseño del módulo.

El diseño de ABP utilizado mantiene dos de los rasgos esenciales de la filosofía del enfoque ABP: (1) el conocimiento se origina como consecuencia del problema planteado, durante el proceso de búsqueda de una solución, (2) se realiza mediante el trabajo en grupo. Además del hecho de que se trata de una aplicación individual del ABP, otra diferencia con respecto al ABP original consiste en que, en nuestro caso, no se puede hablar de un aprendizaje verdaderamente autodirigido ya que el problema se presenta estructurado en subproblemas que orientan el proceso de resolución. En cualquier caso, se trata de una alternativa que algunos expertos en ABP aconsejan en las etapas iniciales del currículo (Albanese, 2010) o cuando los estudiantes no están preparados para afrontar la resolución de problemas (Restrepo, 2005; Branda, 2009)

Los objetivos de la investigación se centraron en comprobar si la utilización del ABP produce mejores resultados en la comprensión de la materia de estudio, en la capacidad de comunicación del conocimiento, en la motivación y en la implicación del alumno en su propio proceso de aprendizaje, en comparación con los métodos de enseñanza más centrados en la enseñanza por parte del profesor.

\section{CONTEXTO ACADÉMICO DE LA INVESTIGACIÓN}

La experiencia docente que sustenta nuestra investigación se llevó a cabo durante el curso académico 20010-11, en el marco de la asignatura Cartografía básica del primer curso del grado de Geografía y Ordenación del Territorio. Se trata de un nuevo grado, diseñado de acuerdo a los principios de convergencia del Espacio Europeo de Educación Superior, que se deriva de una titulación previa denominada Licenciatura en Geografía. La asignatura se imparte en el primer cuatrimestre del curso $\mathrm{y}$, por tanto, se trabaja con estudiantes recién incorporados a la universidad.

En la Universidad del País Vasco, la mayoría de los nuevos grados se han creado con una estructura curricular tradicional basada en asignaturas. Pese a ello, la entidad universitaria está impulsando la utilización de metodologías docentes habituales en los currículos de tipo integrado. De hecho, la universidad ha diseñado un modelo formativo propio, que aspira a implantar de forma progresiva, basado en el uso de metodologías activas de enseñanza-aprendizaje. Para ello, ha puesto en marcha un programa formativo que garantiza la capacitación de un grupo de profesores y la implementación en el aula de las nuevas metodologías docentes, entre las cuales adquiere un protagonismo importante el ABP. En este contexto se enmarcan la experiencia docente y la investigación asociada que aquí se exponen.

\section{PRINCIPIOS Y CRITERIOS PARA EL DISEÑO DEL MODULO ABP}

Ante la diversidad de propuestas existentes en el ámbito del ABP y la inexistencia de un cuerpo teórico sobre el uso de esta estrategia en situaciones aisladas, vimos la necesidad de una guía que orientara nuestras decisiones sobre el diseño del módulo. Teniendo en cuenta que se trata de una aplicación particular del ABP, consideramos que cuestiones como la estructuración de la secuencia didáctica o el tipo y grado de ayuda que debe proporcionar el profesor debían planificarse de acuerdo a las características particulares de la situación. 
Por ello, decidimos realizar un análisis de las circunstancias particulares en las que se iba a aplicar la experiencia, es decir, de las condiciones que definen las coordenadas del punto de partida y que, indudablemente, serán factores importantes del proceso de aprendizaje. Nos referimos a cuestiones tales como la materia de estudio, el nivel académico de los estudiantes, el tamaño del grupo, los hábitos de estudio y estrategias de aprendizaje, el grado de autonomía en el aprendizaje, la disponibilidad de recursos materiales, las prescripciones de la UPV/EHU en relación a la organización de la actividad docente, etc.

Puesto que en el momento de diseñar el módulo didáctico no se conocían las características de los estudiantes receptores, se trabajó con hipótesis basadas en una experiencia docente de 16 años con estudiantes del primer curso de la titulación de Geografía. De forma complementaria, al iniciar el curso se les pasó a los estudiantes un cuestionario en el que se les solicitaba información detallada sobre hábitos de estudio, sobre el manejo de información y sobre el uso habitual de herramientas informáticas con diferentes propósitos. Aunque para entonces lo esencial del módulo didáctico estaba ya diseñado, la información recogida sirvió para matizar las hipótesis iniciales y para adaptar, en la medida de lo posible, las tareas a las características particulares del grupo.

En cualquier caso, quienes acumulamos una larga experiencia intentando convertir nuestras aulas en talleres de aprendizaje sabemos que se trata de un proceso lento que no depende exclusivamente de la buena voluntad y disposición de los docentes o de la bondad de las metodologías que éstos utilizan. Hay que tener en cuenta que existen inercias y obstáculos que dificultan los cambios. Los jóvenes, por ejemplo, que llegan a la universidad traen consigo una larga experiencia en el sistema educativo y, como consecuencia, opiniones y creencias muy afianzadas con respecto a la enseñanza y al aprendizaje, a su función y a la del profesorado. Las preconcepciones a las que nos referimos constituyen, en ocasiones, un obstáculo difícil de superar cuando se pretende incorporar actividades o procedimientos que no concuerdan con sus creencias, su experiencia previa o sus expectativas. Fernández Martínez et al. (2006), en una revisión de estudios empíricos internacionales sobre el ABP, citan a diferentes autores que encontraron resistencia de los estudiantes ante el ABP debido a las diferencias metodológicas que supone. El análisis e hipótesis previas condujeron a la idea de que la implementación del módulo ABP debía prestar especial atención a la existencia de tres tipos de condicionantes:

\section{Con respecto al perfil de los estudiantes.}

Como confirmó, en gran medida, el análisis de los cuestionarios realizados, los estudiantes que han iniciado los estudios de Geografía presentan, habitualmente, las siguientes características: (a) Bajo nivel de autonomía a la hora de buscar información y de elaborar materiales de estudio. (b) Escaso hábito de utilización de Internet para la búsqueda de materiales de estudio. (c) Muy escasa utilización de técnicas de estudio. (d) Baja capacidad de síntesis, medida a través de la capacidad para integrar la información procedente de diferentes fuentes. (e) Elevado nivel de dependencia para con el profesor. (f) Hábito o tendencia persistente a utilizar el aprendizaje memorístico sin pasar por la comprensión de la información almacenada.

Con respecto a la organización de la actividad lectiva.

La docencia de asignatura de Cartografía básica se organiza en tres sesiones semanales de hora y media de duración a lo largo de un período de 15 semanas. Las actividades que se realicen deben tener en cuenta, por ejemplo, que 2 de las 3 sesiones de la asignatura se imparten en dos días consecutivos de la semana. Esto supone que, entre la sesión del primero de los días y la del segundo, los estudiantes no disponen de un período de tiempo para el estudio o el trabajo personal. Por otro lado, la fuerte carga lectiva, que ocupa todos los días de la semana, deja a los estudiantes poco tiempo para iniciarse en el aprendizaje autónomo. Hablamos de un modelo excesivamente presencial y escasamente tutorizado.

Con respecto a la dotación de material informático.

Las aulas disponibles para la docencia de la asignatura no contaban con ordenadores individuales. Este hecho puede considerarse un obstáculo cuando se trabaja con alumnos escasamente capacitados para la búsqueda y gestión autónoma de información. Obliga a 
renunciar a la búsqueda dirigida y supervisada de información en Internet, actividad esta que nos permitiría observar en el aula las dificultades y obstáculos que los alumnos encuentran.

\section{ESTRATEGIA METODOLOGICA Y ORGANIZATIVA}

Las circunstancias mencionadas aconsejaron el diseño de un módulo didáctico cuyas características fundamentales fueron las siguientes:

a) Se basó en un sentido amplio del concepto de problema, entendido éste como una situación de dificultad en la que nos falta el conocimiento necesario para resolverla. Este enfoque del problema se corresponde con lo que Luis Branda (2009) define como situación problemática. Para este autor, la situación problemática es una estrategia destinada no tanto a la resolución del problema como a la potenciación del aprendizaje autónomo. Según el propio autor, el uso de situaciones problemáticas constituye una estrategia apropiada para el comienzo de la formación de un estudiante en un programa de ABP. En la fase inicial no debe esperarse que el estudiante sea capaz de resolver problemas, ya que su base de conocimiento no es suficiente para hacer esto.

b) El problema que guiaba el módulo se presentó a los alumnos mediante un desglose en subproblemas que estructuraban y guiaban el trabajo más de lo que se habría deseado y de lo que muchos de los expertos en el ABP consideran adecuado (Greening, 1998, Hmelo-Silver, 2004; Peace y Kuhn, 2011). El perfil de los estudiantes, el hecho de que carecieran de un conocimiento previo sobre la materia de estudio y la complejidad de esta última fueron determinantes en la decisión.

El problema se organizó en tres subproblemas cuyos enunciados y planteamientos proporcionaban al alumno una visión panorámica y estructurada de la situación problemática. Los subproblemas, a su vez, se organizaron mediante actividades que proporcionaban pautas en cuanto a la operativa de la acción y a los resultados esperados. Se diseñaron un total de 15 actividades que se diferenciaban en aspectos tales como el tipo de estrategia utilizada para impulsar el logro de los objetivos de aprendizaje, el nivel de estructuración o las habilidades cognitivas que impulsaban.

Algunas de las actividades planteadas podrían considerarse más próximas a lo que algunos autores catalogan de ejercicios: conducían a una solución convergente y su resolución implicaba el uso de reglas y procesos lógicos sencillos. Si atendemos, sin embargo, a uno de los criterios más aceptados para la diferenciación entre problemas y ejercicios, la calificación de las actividades es menos evidente. Se puede decir que se trata de un ejercicio cuando nos encontramos ante una dificultad pero conocemos el procedimiento exacto para alcanzar la meta (Pozo, 1995, 16) Para resolverlo utilizamos técnicas que, generalmente, tenemos automatizadas y que, por tanto, no requieren de un proceso de reflexión. La clave está en el componente de rutina que lleve aparejada la actividad que proponemos a los alumnos. Desde esta perspectiva, ninguna de las actividades diseñadas podrían considerarse simples ejercicios. Una de las actividades que consideramos más sencillas, pero no rutinarias, es la que interrogaba a los alumnos sobre los procedimientos para medir la altitud. La pregunta se les planteó mediante el siguiente texto introductorio:

La medición de la altitud ha sido desde siempre una preocupación importante para el ser humano. La curiosidad en unos casos y la necesidad en otros, han impulsado la búsqueda de procedimientos e instrumentos para calcular, con la mayor precisión posible, la altitud de los objetos sobre la superficie terrestre. Hoy, quizás, nos parezca una cuestión banal porque contamos con instrumentos precisos para calcular la altitud. Si nos detenemos, sin embargo, a pensar en el tema, nos daremos cuenta de que la cuestión exige más de una reflexión. Cuándo decimos que la altitud del Monte Everest de.... ¿por cierto, cuál es su altitud?

La distinción entre ejercicios y problemas o el nivel de estructuración y complejidad que éstos deben tener, son cuestiones ampliamente tratadas en el ámbito de la literatura sobre el ABP. En cualquier caso, no parece una cuestión banal en tanto que el uso de unos u otros activa 
estrategias de resolución diferentes. La autonomía en el aprendizaje, uno de los objetivos que perseguimos al utilizar la metodología ABP solo se vería potenciada cuando utilizamos buenos problemas (Hmelo-Silver, 2004). Ahora bien, como concluye Nápoles Valdés (2005) después de una revisión bibliográfica exhaustiva sobre las diferencias entre ejercicios y problemas, se trata de una cuestión extremadamente compleja. En el mismo sentido resultan significativas las palabras de Pozo $(1994,205)$ cuando habla del "carácter relativo y móvil de la frontera entre ejercicios y problemas" o el análisis de Jonassen y Hung (2008) sobre la diversidad de factores que definen la complejidad de un problema.

Otras de las actividades que integraban el módulo eran más exigentes en lo que se refiere a la iniciativa y planificación que demandaban de los alumnos. Incluso conduciendo a una solución convergente, implicaban el manejo y procesamiento de información procedente de diversas fuentes de modo que, además de fomentar las habilidades de procesamiento de la información, forzaban al trabajo colaborativo al tener que repartir tareas entre los miembros de cada grupo. Este sería el caso, por ejemplo, de la cuarta actividad, cuyo enunciado se formuló como sigue:

Si tenemos en cuenta que los mapas topográficos proporcionan información detallada de las altitudes y formas del terreno, no será difícil imaginar que son muchos los profesionales que usan este tipo de mapas como herramientas de trabajo. De eso se trata en este caso, es decir, de elaborar un listado de profesiones o de áreas de actividad en las que la información que proporcionan los mapas topográficos puede resultar de utilidad. El objetivo es que para cada una de las áreas de actividad o profesiones que identifiquéis, describáis la utilidad que puede tener la cartografía topográfica.

c) Para la realización de las actividades, los 22 alumnos integrantes del curso se dividieron en cinco grupos de tamaño variable entre 3 y 5 participantes. Durante las seis semanas que duró el módulo ABP, todas las sesiones lectivas (18 en total) se realizaron a modo de taller de trabajo. Debido a la disponibilidad de un aula cuyo mobiliario no estaba anclado al suelo, antes de iniciar la sesión de trabajo, los alumnos redistribuían mesas y sillas con el fin de formar una mesa grande para cada grupo de trabajo.

d) Las sesiones en el aula, de hora y media de duración, se dedicaron a la presentación de las actividades, a la aclaración de dudas sobre el procedimiento y los contenidos del trabajo y a la explicación, previa a la realización de la actividad, de conceptos cuya complejidad podría haber constituido un escollo. Una vez que todos los equipos habían comprendido los objetivos de la actividad, se fijaba una fecha para su entrega. El trabajo -uno por grupo- debía ser entregado a través de la plataforma Moodle de la asignatura. Se habilitó para ello un espacio Wiki en dicha plataforma.

e) Mucha de la información que los alumnos podían consultar para abordar la resolución del problema se les proporcionó desde el principio, evitando de este modo que tuvieran que encargarse ellos de la búsqueda o selección de materiales.

f) Un fuerte apoyo de las TIC (Tecnologías de la Información y la Comunicación) para impulsar la colaboración y la cohesión de grupo, para facilitar las tareas de tutorización, retroalimentación y evaluación y, por último, para realizar un control del grado de seguimiento por parte de los alumnos.

La plataforma virtual de la asignatura contó con un foro de dudas, comentarios y aportaciones. Se concibió como una herramienta esencial para el seguimiento y control del proceso de aprendizaje y, dentro de éste, del nivel de implicación y del interés de los alumnos. Mediante este foro, único para el conjunto de la actividad $\mathrm{ABP}$, se pretendía impulsar la colaboración, no ya entre subgrupos, sino entre todos los alumnos del curso. Mediante el foro se pretendía, además, obtener un reflejo de la dinámica de trabajo, de las dudas que surgieran y del nivel de colaboración que se establecía entre los alumnos. 
g) En relación al sistema de trabajo, la secuencia organizativa habitual fue la siguiente: 1 . Presentación de la actividad. 2. Lectura y análisis de materiales. 3. Presentación colectiva de dudas y dificultades para cumplimentar la actividad. 4. Elaboración de la actividad. 5. Subida de materiales a la plataforma Moodle. 6. Corrección por parte de la profesora. 7. Retroalimentación colectiva: análisis en el aula de errores, carencias, etc.

La retroalimentación colectiva se planteó con el fin de impulsar la metacognición. Un estudiante metacognitivo es aquel capaz de reflexionar sobre su propio proceso de construcción del conocimiento y, por tanto, sobre su aprendizaje. (Zabit, 2010) La actividad consistió en una corrección pública (en el aula) de los trabajos realizados, de modo que los estudiantes reflexionaran sobre el proceso de construcción del conocimiento que les llevó a resolver el problema. La actividad, que se realizó manteniendo el anonimato de los trabajos, proporcionó a los alumnos la oportunidad de recibir información y de reflexionar sobre lo que era incorrecto, insuficiente o se podía mejorar.

\section{EVALUACION DEL APRENDIZAJE}

Se realizó una evaluación continua, a través de distintos procedimientos: a) Evaluación de cada una de las actividades realizadas mediante análisis y valoración de los resultados previamente incorporados a la Wiki; b) Evaluación mediante tutorías de la aportación individual al trabajo de grupo; c) Evaluación de la participación individual en el foro; y d) examen final

a) Evaluación de cada una de las actividades realizadas mediante análisis y valoración de los resultados previamente incorporados a la Wiki de la plataforma Moodle destinada al efecto. Las actividades se organizaron, desarrollaron y redactaron a nivel de grupo, es decir, todos los integrantes fueron autores y responsables del trabajo y recibieron, en consecuencia, una valoración común. La evaluación se realizó mediante criterios relacionados con (a) los contenidos: adecuación a los enunciados de la actividad, grado de desarrollo y profundidad, riqueza informativa, precisión conceptual, etc. y con (b) la composición formal y la estructura de los textos: ordenación lógica y coherencia de la secuencia de ideas, utilización de estructuras de ordenación (títulos, subtítulos y apartados), etc.

La comparación de los distintos trabajos realizados por los grupos a lo largo del módulo ABP permitió la realización de un análisis sobre la evolución de la calidad de los mismos en relación a los criterios mencionados. Para ello se utilizó una planilla de observación en la que se anotó, para cada uno de los trabajos y epigráfes analizados, un valor variable entre 1 y 5 , siendo el 1 la nota más baja y el 5 la más alta.

b) Evaluación mediante tutorías de la aportación individual al trabajo de grupo. Las sesiones de tutorías se dedicaron fundamentalmente a conocer la aportación de cada alumno al trabajo del grupo y el modo de organizar las tareas.

c) Evaluación de la participación individual en el foro basada en el análisis de la calidad de las aportaciones realizadas. Se utilizó para ello un conjunto de criterios relacionados con el nivel de comunicación/colaboración que suponía el mensaje y con la profundidad del contenido. Los mensajes se clasificaron en cinco categorías de valor creciente: 1: Respuesta breve, sólo para mostrar la presencia del alumno en el foro. 2: Mensaje para plantear una duda o para sumarse a la duda expresada por otro alumno. 3: El mensaje constituye una respuesta de ayuda a las dudas expresadas por uno o varios compañeros. El alumno emite su opinión. 4: El mensaje constituye una respuesta de ayuda a las dudas expresadas por uno o varios compañeros, pero, además, el alumno expresa su opinión y la fundamenta. 5: El mensaje constituye una respuesta de ayuda a las dudas expresadas por uno o varios compañeros; el alumno expresa su opinión y la fundamenta pero, además, aporta información detallada sobre su fuente de referencia.

d) A final de curso se realizó un examen final que incluyó solo los temas y materiales que no se habían trabajado en el módulo de ABP. Con el fin de poder comparar los resultados de los exámenes con los obtenidos por los alumnos en cursos anteriores se utilizaron preguntas y 
criterios de evaluación prácticamente idénticos. Como veníamos haciendo desde hacía varios años, la respuesta a las preguntas utilizadas exigía la integración de conocimientos adquiridos en contextos distintos y en diferentes momentos del curso. Los criterios de evaluación fueron los mismos que los que se utilizaron en la corrección de los trabajos, detallados anteriormente.

\section{RESULTADOS}

Como indicador de la mejora en la calidad del aprendizaje que ha supuesto la introducción de un módulo ABP en el curso de Cartografía básica de 2010-11 hemos utilizado las notas obtenidas por los estudiantes en el examen final de la asignatura. Los resultados los hemos comparado con los obtenidos en los exámenes finales de los cursos 2007-2008 y 2008-2009. Aunque en los tres cursos analizados, y especialmente en el último, se han utilizado pruebas de evaluación diversas, solo los resultados de los exámenes finales resultan comparables ya que en todos ellos se han aplicado los criterios de evaluación mencionados anteriormente.

En general, las notas de los estudiantes implicados en el ABP han sido más elevadas que las que obtuvieron, en la misma asignatura, los estudiantes de cursos pasados sin técnicas de ABP, pero con objetivos y criterios de evaluación muy similares.

Tabla 1: Resumen estadístico de los resultados de los exámenes de tres cursos académicos

\begin{tabular}{|c|c|c|c|}
\hline & \multicolumn{3}{|c|}{ CURSO } \\
\hline & 2007-08 & 2008-09 & 2010-11 \\
\hline Número de alumnos & 13 & 16 & 22 \\
\hline Promedio de la nota del examen & 6,15 & 5,26 & 6,85 \\
\hline Desviación estándar & 2,38 & 2,45 & 1,89 \\
\hline Porcentaje de suspensos & $20 \%$ & $41,67 \%$ & $22,73 \%$ \\
\hline Porcentaje de suspensos + no presentados & $38,46 \%$ & $56,25 \%$ & $22,73 \%$ \\
\hline
\end{tabular}

Si observamos la tabla 1, vemos que el promedio de las notas de examen durante el curso 201011 fue superior al de los cursos anteriores. Auque la elevación del promedio es, en sí mismo, un dato relevante, todavía lo son más los resultados sobre el porcentaje de suspensos y de alumnos no presentados. Destacan, especialmente, los elevados porcentajes de alumnos que, durante los cursos 2007-08 y 2008-09, o bien no se presentaron al examen o bien lo suspendieron. Los porcentajes descienden notablemente en el curso 2010-11. Con respecto a este último curso, lo verdaderamente destacable es que se presentaron al examen todos los alumnos matriculados, siendo este un hecho que, en función de nuestra propia experiencia, resulta insólito.

Otros indicadores que refuerzan los resultados anteriores y que nos informan sobre la motivación y la implicación de los alumnos en su proceso de aprendizaje son los siguientes:

a) La asistencia a clase. Del total de los 22 alumnos matriculados en la asignatura, 19 asistieron a clase de forma regular, entregaron todas las tareas en los plazos previamente fijados y participaron de forma activa en las sesiones de clase y en la plataforma Moodle que sustentaba la asignatura.

b) La participación en el foro (tabla 2). El foro de dudas fue uno de los recursos más visitados de la página Moodle de la asignatura. En la tabla 2, que recoge un resumen descriptivo de los datos sobre participación del alumnado en el foro, podemos observar que recibió un total de 1.855 entradas. Cabe destacar que sólo 2 de los 22 alumnos accedieron únicamente en tres ocasiones a dicho foro. Aunque la variabilidad fue importante, los datos del primer y tercer cuartil muestran que, en cualquier caso, el número de consultas fue, en general, elevado.

Los resultados del análisis de los mensajes depositados mostró que los alumnos hicieron 102 aportaciones al foro y que dichas aportaciones fueron realizadas por 14 de los 22 participantes. De las 102 aportaciones, 18 pertenecían a la categoría $n^{0}$ 1, es decir, al tipo de mensajes breves 
destinados casi exclusivamente a mostrar la presencia del alumno en el foro. Los 84 mensajes restantes fueron preguntas o respuestas a las preguntas efectuadas por sus compañeros. Finalmente, sólo tres de los alumnos emitieron mensajes que incluían una fundamentación más o menos detallada de sus respuestas.

Tabla 2: Datos descriptivos de la participación de los alumnos en el foro de dudas

\begin{tabular}{|l|c|}
\hline Promedio de visitas & 88,3 \\
\hline Desviación estándar & 70,2 \\
\hline Mínimo de visitas & 3 \\
\hline Máximo de visitas & 252 \\
\hline Primer cuartil. Q1 & 34 \\
\hline Tercer cuartil. Q3 & 121 \\
\hline Total de entradas en el foro & 1.855 \\
\hline
\end{tabular}

En relación al análisis de la evolución que mostraron los alumnos del curso 2010-11 desde el inicio del módulo ABP hasta su finalización, realizado mediante comparación de la calidad de los distintos trabajos elaborados por los grupos a lo largo del módulo, los resultados muestran que solo uno de los grupos no experimentó ninguna mejora significativa con respecto a los parámetros analizados. En todos los trabajos, el promedio de evaluación obtenido se mantuvo entre los valores 1 y 2 . Dos de los grupos mostraron mejoras significativas en los aspectos relacionados con los contenidos, en los que el promedio, aunque sufrió variaciones, mostró una tendencia ascendente hasta colocarse en valores entre 3 y 4 . Por último, los trabajos de los dos grupos restantes evidenciaron una evolución positiva importante, tanto en lo que se refiere a los contenidos como a la composición formal y estructura de los textos. En este caso, los valores de la evaluación en ambos apartados superaron el 4 en los últimos trabajos.

\section{DISCUSION}

Aunque resulta innegable que la aplicación del ABP como experiencia puntual en un currículo de estructuración clásica ha producido resultados beneficiosos, surgen muchas dudas con respecto a los factores concretos que más han podido influir en los resultados positivos.

La implementación del módulo $\mathrm{ABP}$ ha supuesto la introducción de numerosos recursos de aprendizaje y de evaluación que no son habituales en la enseñanza tradicional y que pueden haber contribuido en diferente medida a la mejora. Por ello, nos preguntamos, por ejemplo, cuál es el peso que puede haber tenido el uso de problemas, o su desglose en actividades. Nos inquieta, igualmente, saber en qué medida ha podido influir el seguimiento que supone la realización de diversas pruebas de evaluación o de actividades de retroalimentación, o la realización de las sesiones de debate o el trabajo en grupo. Cabe preguntarse, también, qué parte de la mejora puede relacionarse con los recursos y facilidades ofrecidos por la plataforma virtual, o cuáles habrían sido los resultados si hubiéramos añadido la tarea de búsqueda de la información. Consideramos que la incertidumbre sobre la posible influencia de los distintos factores, que ya ha sido señalada y tratada por otros autores (Pease y Khun, 2011), es una cuestión importante que debe orientarnos en investigaciones futuras sobre el $A B P$

Otra de las cuestiones que merecen un análisis y discusión se relaciona con los inconvenientes de la implementación del ABP en contextos de currículos no integrados. En este sentido, queremos destacar el hecho de que el uso de modelos de enseñanza-aprendizaje como el utilizado supone una tarea muy costosa en tiempo y extremadamente compleja. La realización del diseño y validación del mismo $\mathrm{y}$, de forma particular, el seguimiento de la actividad durante su implementación, entrañan un volumen de tabajo materialmente inviable para el profesorado. Para 
entender esta afirmación hay que tener en cuenta que la carga docente habitual del profesorado de la UPV/EHU es de 3 o más asignaturas por año. Este hecho ha sido señalado como un inconveniente de la metodología por diferentes autores. (Tarazona, 2005; Campo et al., 2009) Consideramos imprescindible abordar la creación de equipos docentes y la coordinación del profesorado.

\section{CONCLUSIONES}

De acuerdo a los resultados obtenidos es posible extraer las siguientes conclusiones: 1. La utilización del ABP en contextos curriculares no integrados produce resultados de aprendizaje superiores a los que se obtienen mediante enfoques de enseñanza-aprendizaje más tradicionales, es decir, centrados en la actividad del profesor; 2. La implicación y motivación de los alumnos, medidas a través del grado de cumplimiento de tareas, de la regularidad en la asistencia a clase y de su presentación al examen, se incrementa cuando se aplican estrategias de enseñanzaaprendizaje propias del enfoque ABP; 3 . El uso de un enfoque ABP que integra procedimientos de evaluaciòn continuada y de retroalimentación, produce una evolución positiva observable de la calidad del aprendizaje; y 4. El diseño e implementación del enfoque ABP exige al profesorado una dedicación horaria absolutamente insostenible dentro del actual modelo universitario español.

\section{AGRADECIMIENTOS}

La investigación ha sido financiada por el Vicerrectorado de Calidad e Innovación Docente de la Universidad del País Vasco, a través de los proyectos de Innovación Docente (código 6241)

\section{REFERENCIAS}

Albanese, M.A. Problem-Based Learning. In W. B. Jeffries \& K. N. Huggett (Eds.). An Introduction to Medical Teaching (41-53). Netherlands: Springer. (2010)

Branda, L.A., El aprendizaje basado en problemas: De herejía artificial a res popularis. Educación. Médica. [online]. 12, (1), 11-23 (2009) http://dx.doi.org/10.4321/S1575-18132009000100004.

Campo, J. y otros seis autores, Aplicación de un sistema mixto de Enseñanza Tradicional/Aprendizaje Basado en Problemas en la asignatura de Urgencias en Odontología. Revista Complutense de Educación, 20 (1), 135-150 (2009).

Casado Ortiz, R., Convergencia con Europa y cambio en la universidad. Edutec: Revista Electrónica de Tecnología Educativa. 20, 1-24 (2006)

Dochy, F.; Mien Segers, M., Piet y Van den Bossche, P., Effects of Problem-based learning: a meta-analysis. Learning and Instruction 13, 533-568 (2003)

Fernández Martínez, M. y otros 4 autores, El aprendizaje basado en problemas: revisión de estudios empíricos internacionales. Revista de Educación, 341, 397-418. (2006)

Greening, T., Scaffolding for Success in Problem-Based Learning. Medical Education Online (1998) [serial online], http://www.med-ed-online.org/f0000012.htm. Acceso: 10 de julio de 2010.

Hmelo-Silver, C.E., Problem-Based Learning: What and How Do Students Learn? Educational Psychology Review, 16 (3), 235-266 (2004)

Jonassen, D. H. y Hung, W., All Problems are not equal: Implications for Problem-Based Learning. Interdisciplinary Journal of Problem-based Learning, 2 (2) 4 (2011).

Lee, Y.; Mann, K.V. y Frank, B.W., What drives students' self-directed learning in a hybrid PBL curriculum. Advances in Health Sciences Education, 15(3), 425-437 (2010) 
Levia, Del F., J.R. y Quiring, S. M., Assessment of student learning in a hybrid PBL capstone seminar. Journal of Geography in Higher Education, 32(2), 217-231 (2008)

Lobato, C. y Medinabeitia, A., Perfiles Motivacionales del Profesorado ante la Formación en Metodologías Activas en la Universidad. Formación Universitaria, 4(1), 37-48 (2011).

Martínez Román, M. A. (Coord.), Aplicación del aprendizaje basado en problemas a la formación en competencias de trabajadores sociales In: Propuestas de diseño, desarrollo e innovaciones curriculares y metodología en el EEES. Universidad de Alicante, Alicante, Marfil, Alcoy, España, 467-484 (2009)

Maudsley, G., Do we all mean the same thing by "problem-based learning"? A review of the concepts and a formulation of the ground rules. Academic Medicine, 74: 178-85 (1999)

Michavila, F., La innovación educativa: oportunidades y barreras. ARBOR Ciencia, Pensamiento y Cultura 1, 3-8. (2009).

Mitchell, J.E. y Smith, J., Case Study of the introduction of Problem Based Learning in Electronic Engineering. International Journal of Electrical Engineering Education, 45(2), 131-143 (2008)

Ministerio de Educación y Ciencia. (MEC), Propuestas para la renovación de las metodologías educativas en la universidad. Secretaría de Estado de Universidades e Investigación. Consejo de Coordinación Universitaria, Madrid (2006)

Morales Gil, F. J.; Pardo Rojas, A. y Álvarez Carpio, B., El proceso de convergencia europea en la universidad española, un proyecto en la encrucijada. XXI, Revista de Educación, 12, 15-27 (2010)

Nápoles, J., Aventuras, venturas y desventuras de la resolución de problemas en la escuela. (2005). http://www.edutecne.utn.edu.ar/napoles-valdes/problemas.oz.pdf. Acceso: 3 de marzo de 2010.

Newman M. J., Problem-based learning: an introduction and overview of the key features of the approach. Journal of Veterinary Medical Education, 32(1), 12-20 (2005)

Pease, M.A. y Kuhn, A., Experimental Analysis of the Effective Components of Problem-Based Learning. Science Education, 95 (1) 57-86 (2011)

Pozo, J. I., La solución de problemas como contenido procedimental de la educación obligatoria. In: La solución de problemas. Aula XXI. Santillana: Madrid, p. 180-214. (1994)

Pozo, J. I., Postigo, Y. y Gómez-Crespo, M. A., Aprendizaje de estrategias para la solución de problemas de ciencias, Alambique, 5, 16-26. (1995)

Restrepo, B., Aprendizaje basado en problemas (ABP): una innovación didáctica para la enseñanza universitaria. Educación y Educadores, 8, 9-19 (2005).

http://palabraclave.unisabana.edu.co/index.php/eye/article/view/306/408. Acceso: 12 de mayo de 2008.

Sáez Pérez, L. A., Propuestas para la renovación de las metodologías educativas en la Universidad. Revista electrónica sobre la enseñanza de la Economía Pública, 4, 65 - 73 (2008)

Tarazona, J. L., Reflexiones acerca del aprendizaje basado en problemas (ABP). Una alternativa en la educación médica. Revista Colombiana de Obstetricia y Ginecología, 56 (2), 147-154 (2005)

Zabit, M.M., Problem-Based Learning On Students "Critical Thinking Skills In Teaching Business Education In Malaysia: A Literature Review. American Journal of Business Education 3 (6), 19-32 (2010). 